\title{
Probing dynamic fluorescence properties of single and clustered quantum dots toward quantitative biomedical imaging of cells ${ }^{\dagger}$
}

\author{
Hyeong-Gon Kang, ${ }^{1}$ Fuyuki Tokumasu, ${ }^{2}$ Matthew Clarke, ${ }^{1}$ Zhenping \\ Zhou, ${ }^{1}$ Jianyong Tang, ${ }^{1}$ Tinh Nguyen ${ }^{1}$ and Jeeseong Hwang ${ }^{1 *}$
}

\begin{abstract}
We present results on the dynamic fluorescence properties of bioconjugated nanocrystals or quantum dots (QDs) in different chemical and physical environments. A variety of QD samples was prepared and compared: isolated individual QDs, QD aggregates, and QDs conjugated to other nanoscale materials, such as single-wall carbon nanotubes (SWCNTs) and human erythrocyte plasma membrane proteins. We discuss plausible scenarios to explain the results obtained for the fluorescence characteristics of QDs in these samples, especially for the excitation time-dependent fluorescence emission from clustered QDs. We also qualitatively demonstrate enhanced fluorescence emission signals from clustered QDs and deduce that the band 3 membrane proteins in erythrocytes are clustered. This approach is promising for the development of QD-based quantitative molecular imaging techniques for biomedical studies involving biomolecule clustering. @ 2009 John Wiley \& Sons, Inc. WIREs Nanomed Nanobiotechnol 20102 48-58
\end{abstract}

\begin{abstract}
A variety of biocompatible nanocrystals has been developed as novel nanoscale probes to achieve quantitative optical imaging and diagnostics for assorted biophotonic applications. ${ }^{1,2}$ Among other techniques, success in the development of photostable bioconjugated fluorescent colloidal semiconductor nanocrystals or quantum dots (QDs) has significantly advanced quantitative measurement capabilities for numerous bio-imaging and bio-detection assays, including fluorescence imaging, optical diagnostics of in vivo biological processes, and detection of pathogens in environmental and clinical samples. ${ }^{3-5}$

Although QDs have many unique optical characteristics that enable sensitive and stable optical measurements, the use of QDs as optical probes for quantitative imaging at the molecular level in

\footnotetext{
*Correspondence to: jch@nist.gov public domain in the Unites States of America.

${ }^{1}$ National Institute of Standards and Technology, 100 Bureau Drive, Gaithersburg, MD 20899, USA Twinbrook Parkway, Bethesda, MD 20892, USA

DOI: $10.1002 /$ wnan.062
}

${ }^{\dagger}$ This article is a U.S. Government work, and as such, is in the

${ }^{2}$ National Institute of Allergy and Infectious Diseases, 12735
\end{abstract}

complex biological environments is still challenging as key optical properties, such as quantum yield and fluorescence emission, are significantly influenced by variations in the QD nanoscale environment. ${ }^{6,7}$ Therefore, optical characterization of single or clustered QDs in controlled or known physical and chemical environments is of exceptional importance in understanding the mechanisms affecting the optical properties of QDs. For instance, variations in physicochemical conditions at the surface of QDs (e.g., band gap or thickness of a surface passivation layer in binary semiconductor QDs, ionic strength of surface-conjugated organic molecules in water-soluble QDs, etc.) are introduced in a controlled fashion to evaluate their influences on the optical properties. ${ }^{8}$ For real applications, nanotechnology to engineer QDs has enabled a variety of measurement strategies to use QDs as functional optical elements and devices such as nanobiosensors. ${ }^{5,9-12}$

For example, single colloidal QDs in solution have been employed to probe their immediate nanoscale environment such as ionic strength or $\mathrm{pH} .{ }^{13,14}$ In addition, the fundamental mechanisms of QDs' interactions with other nanoscale materials have 
been investigated. ${ }^{15}$ To this end, recent reports have shown that self-interacting QDs in aggregate or QDs interacting with other solid-state nanoscale materials, such as single-wall carbon nanotubes (SWCNTs) and metallic nanoparticles, modify the optical characteristics of QDs. ${ }^{16-18}$ This approach has enabled the assessment and control of nanoscale assembly processes involving QDs and other nanoscale materials. ${ }^{19}$

Among other optical characteristics of QDs, fluorescence properties such as blinking and lifetime have been used to assess the nanoscale environment characteristics and to develop optical sensor platforms to investigate chemical and biological processes. A fundamental understanding of the fluorescent characteristics of these surface-functionalized QDs in different physical or chemical conditions is essential for their use in quantitative molecular imaging for biological and biomedical applications. Here, we discuss how the emitted fluorescence is modified when surface-functionalized single QDs are bound to SWCNTs or aggregate when linked to clustered proteins in the cell membrane. To demonstrate the plausibility of quantitative molecular imaging of real biological samples, we present our preliminary results on the enhancement of fluorescence emission from clustered QDs in human erythrocytes. Further development of this approach will enable quantitative optical characterization of target molecules in single cells to illuminate their physical, chemical, and biochemical processes. ${ }^{20-22}$

\section{EXPERIMENTAL}

\section{Fluorescence Intermittency of Single and Clustered QDs}

QD samples were prepared on clean Corning borosilicate glass coverslips obtained from Ted Pella (Redding, CA). Prior to use, the coverslips were cleaned in piranha solution $\left(100 \% \mathrm{H}_{2} \mathrm{SO}_{4}: 30 \% \quad \mathrm{H}_{2} \mathrm{O}_{2}\right.$, 3 : 1 volume) at room temperature for $24 \mathrm{~h}$, rinsed thoroughly with $18.2 \mathrm{Mohm}$ water from a Nanopure Diamond Life Science Water Purification System (Barnstead International, Dubuque, IA) and stored under water. Coverslips were dried under a stream of nitrogen just prior to use followed by $20 \mathrm{~min}$ of ultraviolet-ozone cleaning in a Jelight ozone oven (Irvine, CA). Approximately $3 \mu \mathrm{L}$ of nominally $10 \mathrm{nmol} / \mathrm{L}$ or $1 \mu \mathrm{mol} / \mathrm{L}$ QD solutions with carboxyl-functionalization or streptavidinconjugation (CdSe/ZnS, 588-608 nm emission peaks, Evident Technologies, Troy, NY) were placed on the cleaned glass coverslip and spin-cast to produce spatially isolated QDs. The coverslip was attached to an XYZ closed-loop piezo stage from Mad City Labs (Madison, WI) and positioned directly above a high numerical aperture (100X, 1.45 NA) objective lens from Zeiss (Thornwood, NY) mounted on an inverted fluorescence microscope, Zeiss Axiovert 135TV (Thornwood, NY). The $488 \mathrm{~nm}$ line from an argon ion laser was directed through a holographic band pass filter from Kaiser Optical Systems (Ann Arbor, MI), coupled into the objective lens via the back port of the microscope, and focused directly above the coverslip. The QD sample in air was rasterscanned through the diffraction-limited laser focus and the fluorescence emission from the sample was collected with the same objective lens. The emission light was filtered to remove residual laser excitation using a $488 \mathrm{~nm}$ holographic notch filter from Kaiser Optical Systems (Ann Arbor, MI) and a 515-nm long pass filter from Chroma Technology Corp (Rockingham, VT), and imaged onto an avalanche photodiode detector (APD) type SPCM-200 (EG\&G, now Perkin Elmer, Fremont, CA). Images were constructed pixel by pixel by stepping the piezo stage in $50 \mathrm{~nm}$ increments using a $10 \mathrm{~ms}$ dwell time at each pixel. From the collected fluorescence image, the coordinates of individual QDs were recorded and used to position these QDs at the center of the focused laser spot for further analysis. Individual QDs or clusters were then exposed to continuous laser excitation $(5 \mu \mathrm{W}$ at $488 \mathrm{~nm}$ ) and the fluorescence emission was collected for $10 \mathrm{~min}$ periods with $8 \mathrm{~ms}$ time resolution. After collecting this fluorescence signal, the sample was reimaged to ensure that no significant drift of the sample occurred during data collection (data not shown). All aspects of the imaging and data collection process were controlled using a RHK Technology Inc. (Troy, MI) controller and data acquisition software.

\section{Conjugation of QDs and SWCNTs}

Purified high-pressure $\mathrm{CO}$ conversion $\left(\mathrm{HiPCO}^{\mathrm{TM}}\right)$ SWCNTs were obtained from Carbon Nanotechnologies, Inc., and used as received. The preparation of DNA-wrapped SWCNTs (DNA-SWCNTs) is described in detail elsewhere. ${ }^{23,24}$ Briefly, SWCNTs were sonicated in buffer solution $(200 \mathrm{mmol} / \mathrm{L} \mathrm{NaCl}$ in de-ionized (DI) water, $100 \mathrm{mmol} / \mathrm{L}$ Tris buffer, $5 \mathrm{mmol} / \mathrm{L} \mathrm{NaN} \mathrm{N}_{3}$ solution buffered to $\mathrm{pH} 7$ with $\mathrm{HCl}$ ) in the presence of 30 -mer $5^{\prime}$-GT $(\mathrm{GT})_{13} \mathrm{GT}$ $3^{\prime}$ single-stranded DNA, followed by centrifugation at $21,000 \mathrm{~g}$ for $2 \mathrm{~h}$. The resulting supernatant is a stable, black liquid containing well-dispersed SWCNTs. COOH-functionalized QDs (Invitrogen) were conjugated to the DNA-SWCNTs. The conjugation procedure is similar to that described previously by 
Banerjee et al. ${ }^{25,26}$ and the characterization of the conjugation steps is described in detail elsewhere. ${ }^{27}$ After conjugation, the sample was washed, centrifuged, and decanted twice. The final concentrated solution was resuspended in DI water for optical microscopy.

\section{Differential Interference Contrast Microscopy and Fluorescence Microscopy of the Conjugates of Single-wall Carbon Nanotubes and QDs}

Differential interference contrast (DIC) microscopy was accomplished using a tungsten lamp with a 0.55 NA condenser on an Olympus IX-81 inverted microscope with polarizers and Nomarski prisms (Olympus America, Center Valley, PA) using a 1.45 NA PlanAPO total internal reflection fluorescence oil objective. For epi-fluorescence imaging, a $457 \mathrm{~nm}$ band pass filter was used to select a small spectral range from the $\mathrm{Hg}$ arc lamp. The optical signal was imaged onto an electron multiplying charged coupled device (EM-CCD) camera (Andor Technology, Ireland). The lifetime image of QDs conjugated to SWCNTs was achieved by diffraction-limited confocal scanning microscopy with a Ti:Sapphire laser (76 MHz) excitation at a frequency-doubled wavelength of $459 \mathrm{~nm}$. A pulse picker (NEOS Technologies, Melbourne, FL) was employed to reduce the repetition rate to $7.6 \mathrm{MHz}$. The excitation laser power was about $14 \mu \mathrm{W}$ with a dwell time of $10 \mathrm{~ms}$ per pixel. The lifetime for each pixel was obtained by a data acquisition (DAQ) Becker-Hickel SPC830 DAQ board (Sync In mode) to construct an image of $128 \times 128$ pixels.

\section{Fluorescence Microscopy of Clustered QD Thin Films and QD-labeled Human Erythrocytes}

A droplet of approximately $20 \mu \mathrm{L}$ of a nominal $1 \mu \mathrm{mol} / \mathrm{L}$ solution of streptavidin-coated QDs was placed on a cleaned glass coverslip and dried under a laminar flow hood in a clean room. After drying, the sample was viewed using an Olympus America Inc. (Center Valley, PA) IX81 epi-fluorescence microscope with a $500 \mathrm{~W} \mathrm{Hg}$ arc lamp running at $200 \mathrm{~W}$ with a fluorescence filter set [a bandpass exciter $(447 \mathrm{~nm}$ $\pm 15 \mathrm{~nm}$ ), a dichroic mirror (505 nm cutoff), and a longpass emission filter (560 nm cutoff)], and a 1.25 NA oil-immersion objective]. Images were captured by an intensified, cooled charge-coupled I-PentaMAX camera from Roper Scientific (Duluth, GA). For analysis of the excitation time-dependent fluorescence (ETDF), movie frames of the same area of the sample were taken every $100 \mathrm{~ms}$ and the pixel intensities within a fixed region of interest in each frame were averaged and plotted versus the exposure time. To allow partial exposure of the sample, an aperture iris was placed at the back imaging plane of the objective to focus the field stop aperture onto the sample surface.

Fixation and labeling of band 3 proteins in human erythrocytes with QDs were performed as described previously. ${ }^{28}$ Briefly, the cleaned coverslips were treated with a $2.5 \%(\mathrm{w} / \mathrm{v})$ Alcian Blue solution obtained from Sigma-Aldrich (St. Louis, MO) to achieve sufficient erythrocyte attachment sites. Approximately $0.25 \mathrm{~mL}$ of 0.2 hematocrit erythrocyte suspension in serum-free Roswell Park Memorial Institute (RPMI) medium supplied with $25 \mathrm{mmol} / \mathrm{L}$ of 4-(2-hydroxyethyl)-1piperazineethanesulfonic acid (HEPES) buffer were applied to the coverslips and incubated at $37^{\circ} \mathrm{C}$ for $30 \mathrm{~min}$. Loosely attached erythrocytes were removed by gently rinsing with warmed RPMI, and the remaining erythrocytes were crosslinked with $50 \mathrm{mmol} / \mathrm{L}$ dimethylsuberimidate (DMS) from Sigma-Aldrich in a sodium borate buffer, $\mathrm{pH} 9.5$, containing $1 \mathrm{mmol} / \mathrm{L}$ $\mathrm{MgCl}_{2}$ for $1 \mathrm{~h}$. Erythrocytes were fixed again with $2 \%$ paraformaldehyde (PFA) obtained from Electron Microscopy Science (Fort Washington, PA) in

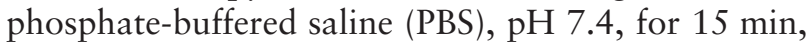
followed by a quenching step with $0.1 \mathrm{~mol} / \mathrm{L}$ glycine in PBS for $1 \mathrm{~h}$ and blocked with $3 \%(\mathrm{w} / \mathrm{v})$ bovine serum albumin (BSA) Fraction V (Sigma-Aldrich) in PBS overnight. After a brief permeabilization with $0.2 \%$ Tween 20 in PBS containing 3\% BSA, erythrocytes were reacted with antihuman band 3 monoclonal antibodies (1/3000 dilution, Sigma-Aldrich) for $1 \mathrm{~h}$ at room temperature. Erythrocytes were washed thrice with the PBS/Tween solution for $5 \mathrm{~min}$ to remove nonspecifically associated IgGs, followed by incubation for 30 min with anti-mouse IgG goat secondary antibody conjugated with biotin (Sigma-Aldrich) and then washed with the PBS/Tween solution to reduce the background signal from unbound secondary IgG molecules. Erythrocytes were then exposed to 10-20 $\mathrm{nmol} / \mathrm{L}$ of streptavidin-conjugated QD585 from Invitrogen (Carlsbad, CA) for 30 min and washed thrice with PBS/Tween to remove nonconjugated QDs.

\section{RESULTS AND DISCUSSIONS}

Optical characterization of individual colloidal QDs with diffraction-limited confocal microscopy requires sufficient dispersion of QDs on a substrate with not more than one QD within the focal volume. Spin coating of QDs on hydrophilic glass substrates from a solution with low concentrations $(<1$ 
FIGURE 1 | (A, B)

Confocal fluorescence images of QDs on glass substrates spin casted from low concentration $(A)$ and high concentration (B) QD solutions. Arrow marked positions, (a), (b), and (c) in the images are the positions from which the time trace of fluorescence intensities presented in (C), (D), and (E) are measured, respectively. Inset in $(A)$ is a magnified view of the area over position (a) exhibiting the 'blinking' behavior of a single QD.

$\mathrm{nmol} / \mathrm{L}$ ) of QDs routinely produced sufficiently low coverage of single QDs to allow imaging and analysis of individual QDs with diffraction-limited confocal techniques (Figure 1A). The image of a single mercapto-undecanoic acid -functionalized QD (Figure 1A, arrow ' $a$ ') includes several pixels in a single scan line due to the convolution between the physical dimension of the QD, the focused laser beam diameter, and the XYZ stage step size. This image demonstrates fluorescence intermittency, indicative of emission from a single QD along individual scan lines and across different scan lines (Figure 1A inset). To further investigate this variation in fluorescence intermittency, individual QDs were positioned at the center of the focused laser beam, and the fluorescence emission was measured under continuous excitation for $40 \mathrm{~s}$ time periods with $10 \mathrm{~ms}$ time bins. The fluorescence intensity from the same single QD shown in Figure 1C demonstrates the intermittency phenomenon in which the QD either emits fluorescence (on) or is nonfluorescent (off). The on/off transitions clearly exhibit quantized steps between fluorescence emission and background levels confirming that the emission is from a single QD. Although most fluorescent spots in these samples exhibit characteristic quantized 'blinking' behavior of single QDs, brighter fluorescent spots deficient of this quantized intermittency are occasionally observed (Figure 1A, arrow ' $\mathrm{b}$ '). As shown in Figure 1D, a typical time trace of the emission from the bright spot exhibits characteristics of multiple QDs, with photon counts an order of magnitude higher. The time trace shows relatively larger intensity fluctuations than that of single QDs. This is a result of the summation of stochastic fluctuations of independent quantized blinking of multiple yet isolated QDs within
$(\mathrm{Hz})$
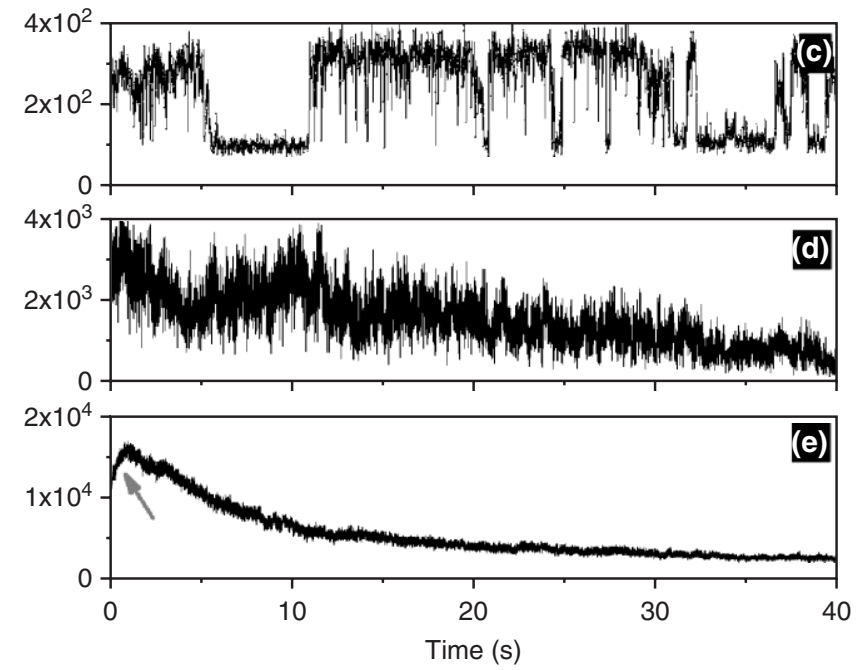

the confocal volume. Upon increased laser intensity, the stepwise decrease of fluorescence intensity due to a series of quantized photo bleaching of QDs also confirms the existence of multiple QDs in the confocal volume.

Confocal scanning microscopy of spin-cast streptavidin-coated QDs onto a glass substrate from a highly concentrated QD solution $(>100 \mu \mathrm{mol} / \mathrm{L}$ ) frequently results in even brighter fluorescent patches in the image. The maximum fluorescence emission intensities within these patches are about two orders of magnitude higher than those of single QDs (Figure 1B). These high fluorescence intensities and irregular, non-Gaussian patchy patterns imply that QDs exist in clustered forms when surface-conjugated proteins induce aggregation of multiple QDs. The time trace of the emission intensity shown in Figure 1E, measured from a point within the bright patch (Figure 1B, arrow ' $c$ '), show characteristics which are not seen from single QDs and low density groups of QDs obtained from dilute QD solutions. The most noticeable difference consistently observed from these aggregated QDs is an emission intensity increase early in the time trace. We observed that the rate and maximum peak intensity of this initial increase vary and depend upon experimental conditions, such as the QD solution concentration, the shelf life of the solution before spin casting, the excitation power of the confocal beam, and the type of surface functionalization. The initial emission intensity increase is followed by gradual decrease until complete photo bleaching of all the QDs occurs.

The blinking phenomenon observed in isolated QDs is attributed to exciton separation and recombination kinetics. ${ }^{3,29-31}$ Absorption of excitation light by a QD results in the creation of an exciton in the 
QD in which a charge carrier is likely ejected from the QD core to trap states on or near the QD surface to produce an ionized, nonemitting QD. This excited charge carrier can follow two possible pathways. The first pathway is to return to the valence band and recombine with the counter charge (electron or hole) to emit a photon. As Figure 2A illustrates, the second pathway is for the exciton to localize at a surface trap state via Auger-assisted ejection or tunneling to result in a quasi-stable dark state of an ionized QD. The excitonic charge carrier undergoing ionization transitions results in a nonfluorescent QD likely trapped in two states (at an extremely fast rate or through a quasi-stable dark state) that produce distinct optical characteristics. In contrast, extended nonemissive states of QDs are the result of charge carrier transitions to deep trap states such as defects on the surface of the QD, or to the substrate or functional coatings at the QD surface. For instance, our previous study of single QDs demonstrated that surface conjugation with electron-donating thiolate groups significantly lowers the rate of charge ejection to deep trap states, and/or conversely, increases the rate of charge return to the QD to result in higher quantum yield than in QDs conjugated with other organic ligands providing highly populated and complex deep trap states. ${ }^{6}$

On the other hand, for QDs in contact with each other within an aggregate, a charge ejected from a QD to the surface trap state can be attracted to a neighboring QD ionized with an opposite charge (Figure 2B). This increased population of counter charges results in the radiative recombination of an excitonic charge pair at a higher rate than the recombination rate in single photoexcited QDs. According to this ejected charge exchange (ECE) model, when clustered QDs are exposed to constant excitation, the 'off' time period that the ejected charges are trapped in deep trap states is shortened; therefore, the quantum yield or fluorescence photon counts per bin time increases. This may result in an initial increase in the time trace of the fluorescence intensity (Figure 1E). The quantum yield of clustered QDs under constant illumination increases toward its maximum value until the system reaches a quasi-equilibrium state with maximum populations balanced between the number of ejected charges and ionized QDs. As QDs continue to be exposed to the excitation light, the quantum yield begins to drop. This is due to the growth of a photo-oxidized layer in QDs, resulting in a buildup of tunneling barriers and subsequent decrease of the ECE rate among QDs. ${ }^{32,33}$ This photo-oxidation process eventually obscures the quantum confinement structures of excitonic (a)
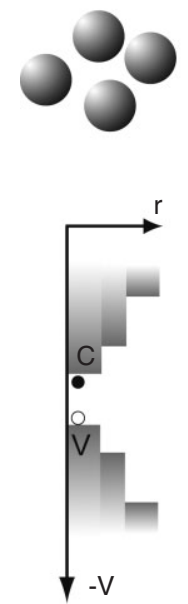

(b)
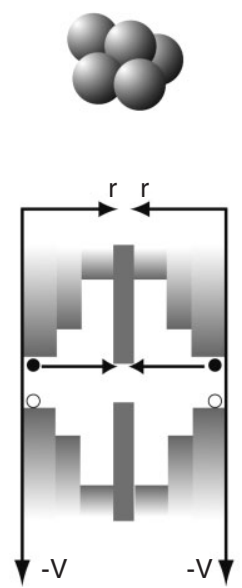

(c)
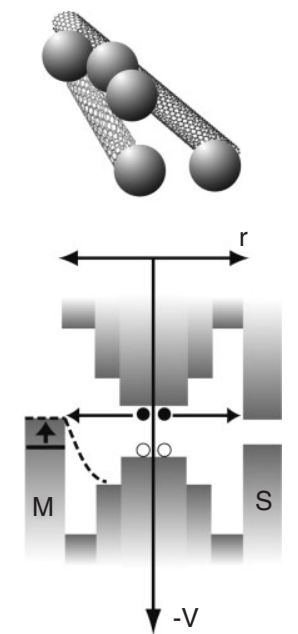

FI G URE 2 | Illustrations of energy states of QDs in different conditions which may result in unique fluorescence emission characteristics from (A) noninteracting, separated single QDs undergoing simple exciton creation/recombination process, (B) interacting QDs in close contact where electrons may be exchanged by tunneling through the contact interface, resulting in increased recombination rates, and (C) QDs in contact with metallic (left side) SWCNTs where the charge flow from an optically excited QD to a metallic nanotube is induced until the thermal equilibrium is reached. (right side) QDs in contact with low-band gap SWCNTs where energy transfer from $Q D$ to nanotube may occur, thereby decreasing the $Q D$ emission.

charge carriers in QDs, resulting in a complete deprivation of their fluorescence emission, in other words, quenching.

We further examined the influence of metallic or low-bandgap nanoscale materials on the optical characteristics of QDs. Upon excitation of QDs conjugated to these materials, the photonic energy creates excitons or excitonic charge pairs in these QDs, but their photoluminescence involves a more complex mechanism than the simple ECE model described above. As Figure 2C illustrates, the annihilation of excitonic charge pairs to convert excited QDs into the neutralized state may be possible through direct exchange of charges between a photoexcited QD and the surrounding material, where the density of states of counter charges (excitonic charges or free electrons in the conjugated materials in contact with the QD) are allowed to contact at the interface. Alternatively, when the fluorescence emission spectra overlaps with the absorption spectra of the conjugated material, Förster resonance energy transfer (FRET) may also be possible through nonradiative energy transfer from a photoexcited donor (QD) to a ground-state acceptor (surrounding material) in proximity of the donor. ${ }^{34-36}$ 
FIGURE 3 | (A) A DIC image of SWCNT-QD hybrid materials in water on a glass coverslip. (B) Fluorescence image of the same sample area. The two time evolution measurements of ETDF intensity of QDs in this sample are plotted in Figure 5D by calculating the average intensity of the pixels within the outlined areas ('a' and 'b') shown on image B. (C, D) A confocal fluorescence emission intensity and fluorescence lifetime micrograph, respectively, of the area are outlined by a box in A, B. Black dots in the lifetime image are most likely due to imperfections of the home-built synchronization electronics.

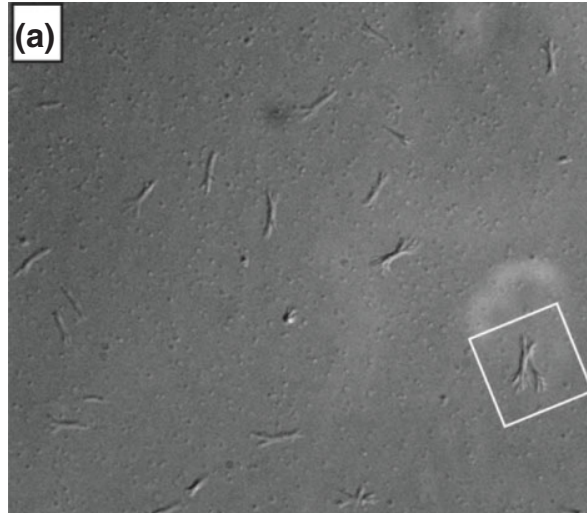

(b) $20 \mu \mathrm{m}$

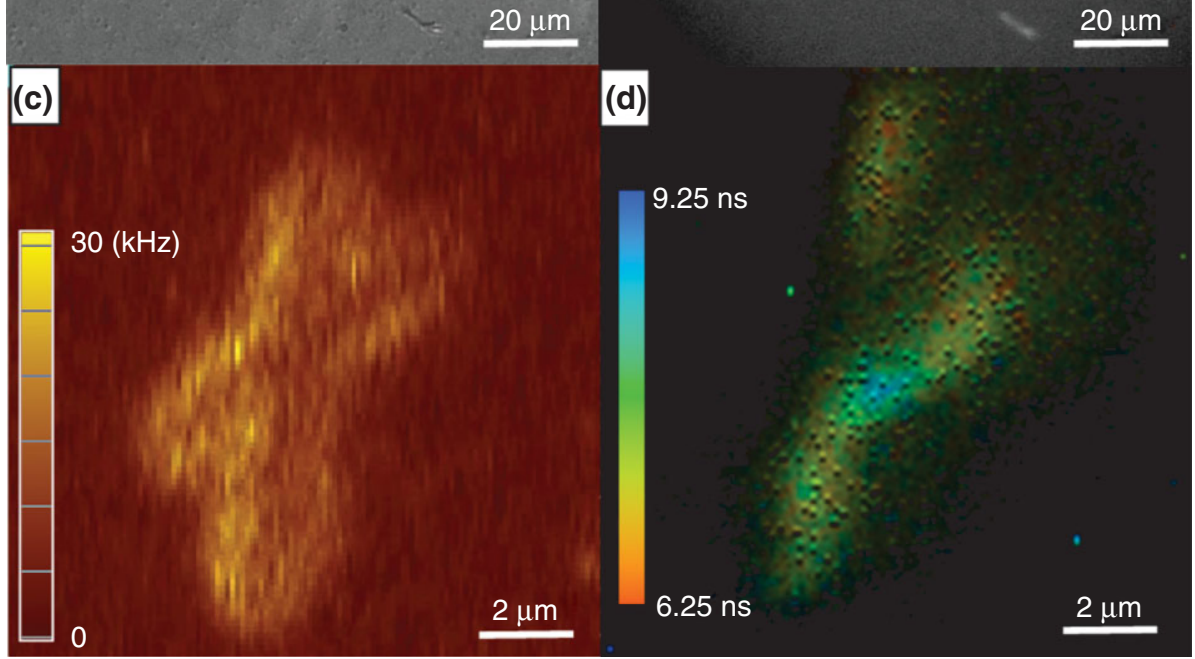

These charge exchange and energy transfer processes involve nonradiative relaxation of photoexcited QDs; therefore, both the photoluminescence and fluorescence lifetime of these QDs are expected to be quenched. ${ }^{16}$

To investigate the phenomenon described above, SWCNTs were conjugated to QDs. Batches of SWCNTs used in this study are a mixture of metallic and semiconducting materials, and they exhibit absorption wavelengths overlapping the emission wavelengths of the QDs. ${ }^{37}$ In brief, we conjugated QDs to dispersed SWCNTs via covalent amidation, employing singlestranded DNA oligonucleotides with guanine $(G)$ and thymine $(\mathrm{T})$ repeating units as linkers between QDs and SWCNTs. The details of this conjugation procedure are described elsewhere. ${ }^{23}$ Figure 3 (A and B) shows a typical DIC micrograph and a fluorescence image of SWCNT bundles conjugated with QDs. DIC imaging allows for visualization of the materials without requiring fluorescence. This technique can provide high contrast images of materials with similar light transmission properties and relies on differences in refractive index. Although analogous images could be obtained through standard transmission microscopy, the DIC images exhibit sharper contrast at the particle borders. Several larger bundled materials are seen in the DIC image (Figure 3A) along with smaller more rounded particles. We compare the DIC image with that obtained by wide-field epifluorescence microscopy (Figure 3B). Although confocal microscopy allows for better spatial resolution (especially along the $z$-axis), it requires scanning of the excitation light across the sample. Here, the entire image is measured simultaneously (by imaging the signal onto an EM-CCD camera) with the drawback of additional out-of-focus signal (as evidenced by the higher background in Figure 3B). From the fluorescence images, we observe that the very small round objects are not fluorescent, while the larger DNA-SWCNT bundles are densely decorated with QDs. Reaction of multiple carboxylic groups from a single QD to two or more DNA-SWCNTs can link many nanoparticles together, resulting in 'bundles' of SWCNTs. This is the likely cause of the large structures observed by DIC and fluorescence. The same conjugation procedure was repeated without 

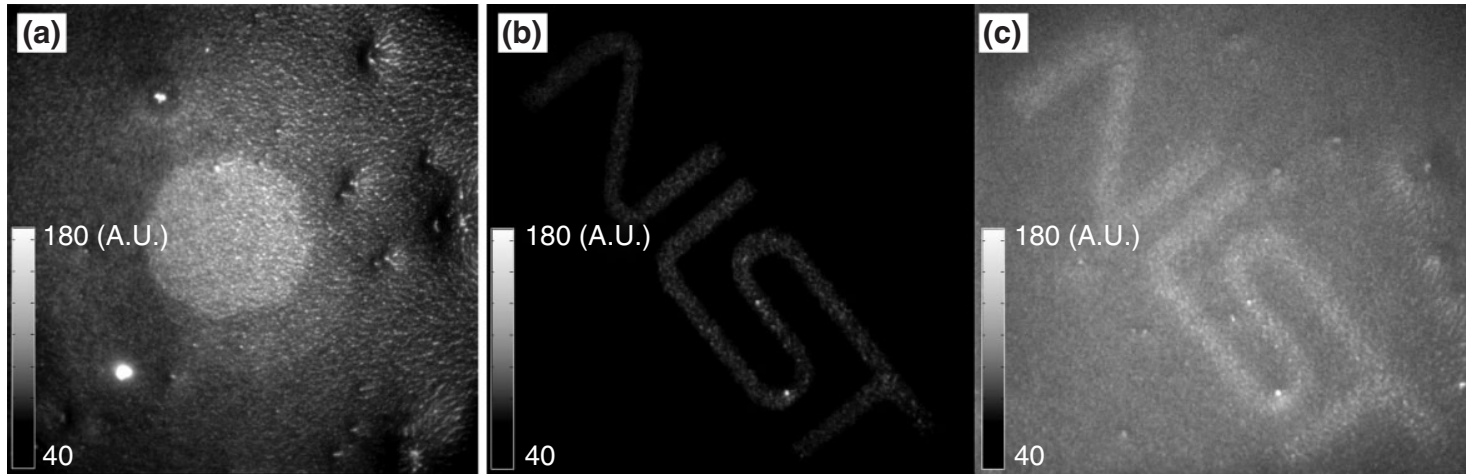

FIG URE 4 | (A) Fluorescence micrographs demonstrating the enhancement in ETDF from a thin film of streptavidin QDs on a glass substrate. After selectively exposing the area of a focused iris aperture to a fluorescence excitation light source for $360 \mathrm{~s}$, the preexposed area of this sample shows significantly enhanced ETDF. (B, C) ETDF micrographs of this sample with excitation light focused onto the sample surface through a letter shaped slot with $(B)$ and without $(C)$ of the mask, exhibiting enhancement of ETDF from the preexposed area of the mask.

linking reagents to estimate the nonspecific interactions between the nanomaterials. No QD-SWCNT coupling was observed in this control experiment (data not shown). A fluorescence confocal image of QD-conjugated SWCNTs (Figure 3C) quantitatively shows the fluorescence intensity distribution from conjugated QDs. Although transmission electron microscopy (TEM) results exhibit relatively uniform distribution of QDs over the entire SWCNTs (data not shown), the fluorescence image shows bright emission only along the SWCNT bundle boundary. Diminished QD fluorescence emission is observed in regions where the SWCNT concentration is high, indicating SWCNTs quench QD photoluminescence. The decreased QD fluorescence lifetime after conjugation to SWCNTs further supports the evidence that nonradiative relaxation of photoexcited QDs becomes dominant when the QDs are conjugated to metallic or low-band gap materials.

However, to quantitatively assess the influence of SWCNT conjugation on the fluorescence quenching of QDs, the optical characteristics of this hybrid sample need to be interrelated with the nanoscale physical and chemical environments, such as the chemical bonding at the interface of the two materials, the density distribution of QDs, and the quantity and composition of conjugated SWCNTs. Here, we report only qualitative results on the effect of SWCNTs by comparing ETDF intensities between samples of SWCNT-conjugated QDs and of proteincoated QDs on glass substrates. Details of the image acquisition and analysis methods are described in the experimental section. Upon photoexcitation, nonradiative charge or energy transfer in the SWCNT-QD system would substantially quench the fluorescence intensity as well as the ETDF intensity of QDs in the thin film, while the ETDF would be much more prominent for aggregated proteincoated QDs in the absence of SWCNTs. Figure 4 shows ETDF results from clusters of protein-coated QDs on glass substrates. To measure the ETDF of QDs, a thin film of streptavidin-coated QDs was prepared on a glass substrate, and a partially open iris field-stopped aperture in front of the illumination light source was imaged onto the middle of the sample surface and was left open for $6 \mathrm{~min}$ to preexpose the QDs within the open aperture area. After this preexposure, the iris field-stopped aperture in front of the illumination light source was removed to image the entire field of view of the sample. The fluorescence image in Figure 4A clearly shows enhanced ETDF of the preexposed QDs. An additional experiment using a letter photomask (Figure 4B and C) to expose a streptavidin-coated QD covered slide exhibited similar results: the preexposed region of QDs is brighter than the unexposed region. On the other hand, the ETDF enhancement of SWCNT-conjugated QDs is not significant, and is approximately 1 order of magnitude less than that observed from aggregated streptavidin QDs (Figure 5D and E). At early exposure times (Figure 5D), the QDs conjugated to SWCNTs show monotonously increasing ETDF. However, the rate of increase is much lower than that of streptavidin QDs at later times.

The ETDF measurement may be used to quantitatively evaluate the self affinity or homooligomerization of proteins in cell membranes. In previous studies involving QD-immunolabeling of band 3 proteins in the plasma membranes of human erythrocytes, significant oligomerization of band 3 proteins was observed after the erythrocytes were infected with the Plasmodium falciparum malaria parasite. ${ }^{38}$ Band 3 oligomerizations are recognized 

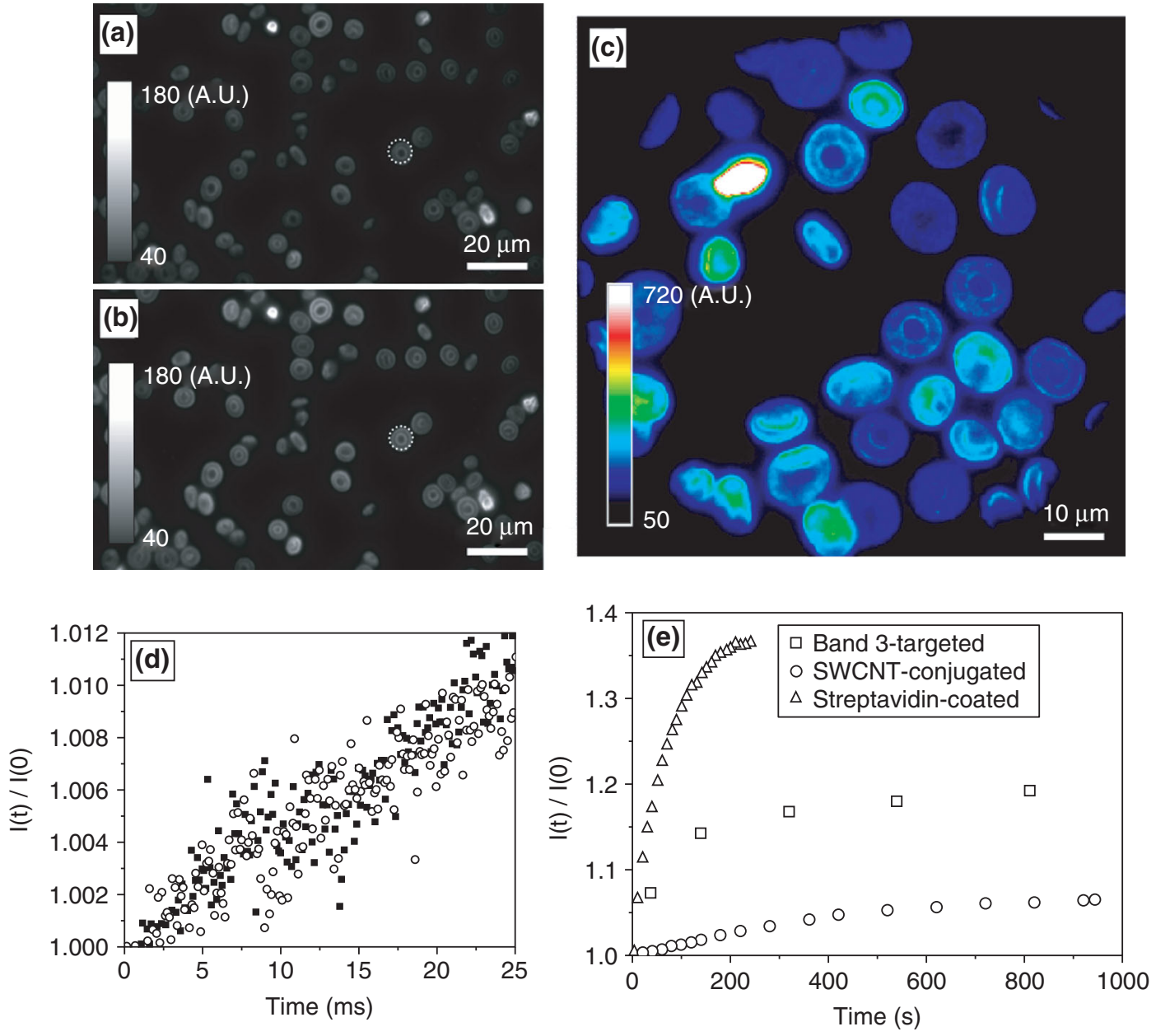

FI G URE 5 | (A, B) Fluorescence images of human erythrocytes with their band 3 transmembrane proteins labeled with anti-band 3 conjugated QDs. Two images from the sample taken at two different continuous exposure times (10 s for image (A), and $800 \mathrm{~s}$ for (B)) are displayed. The corresponding time evolution of ETDF intensity from a single cell is also plotted in (E) by calculating the averaged intensity of pixels within the circular area shown on the image. (C) A fluorescence micrograph of the same batch of QD-labeled human erythrocytes taken at an exposure time of $>1000 \mathrm{~s}$. The variation of the intensity of cells becomes more pronounced as some cells show increased ETDF and some decreased emission intensity due to quenching of some QDs in the cells. (D) The time evolution of ETDF intensity of SWCNT-QD hybrid samples, calculated from the averaged intensity of the pixels within the outlined areas ('a' and 'b') shown in Figure 3B. The signal is normalized to the initial intensity measured from the first frame of the series (zero exposure time). (E) Time evolutions of the ETDF intensities of all three samples, streptavidin QDs, SWCNT-conjugated QDs, and QD-labeled band 3 proteins in erythrocytes, are extracted from a series of movie frames for each sample.

as a result of a significant increase in intracellular oxidation level due to digestions of erythrocyte hemoglobins by parasites as well as the normal senescence process. Oxidated hemoglobins such as methemoglobines continue to degrade and form 'hemichromes' that attach to the cytoplasmic tail of band 3. The attachment of hemichromes is considered as a major driving force of band 3 oligomerization. During the normal erythrocyte recycling mechanism, the oligomerized band 3 seems to be recognized as a 'neoantigen' by host immunoglobulins and stimulates efficient capturing of these erythrocytes. Therefore, developing a method for the sensitive detection of band 3 oligomerization level is critical from an immunology stand point of view. However, common fluorescence microscopy imaging methods used to quantify band 3 clustering required complex mathematical data analyses due to the light diffraction limit. Therefore, ETDF measurements are a suitable alternative for highresolution detection of such small protein clustering. In this study, biochemical assays on nonparasitized erythrocytes show the oligomerization of band 3 proteins in the membrane, although the degree of oligomerization is much less than parasitized erythrocytes. In an attempt to evaluate the oligomerization 
of band 3 proteins in nonparasitized erythrocytes, ETDF measurements were conducted on QD-labeled nonparasitized erythrocytes, where band 3 proteins are immunolabeled with streptavidin-conjugated QDs at a ratio of approximately $1: 1$. As Figures $5 \mathrm{~A}$ and $\mathrm{B}$ demonstrate, the images taken at two different exposure times (10 s for image (A), and $800 \mathrm{~s}$ for (B)) show increases in the ETDF signal for all nonparasitized erythrocytes as the exposure time increases. Comparing this result to that reported above, in which a thin film of concentrated QDs shows enhanced ETDF and single QDs on glass substrates did not yield any measurable ETDF enhancement, we believe that this enhanced ETDF from erythrocytes is a consequence of immunolabeled QDs clustering due to the aggregation of labeled band 3 proteins. Corresponding time evolutions of the ETDF intensities of all three samples, streptavidin QDs, SWCNT-conjugated QDs, and QD-labeled band 3 proteins in erythrocytes, are extracted from a series of movie frames for each sample, normalized to the zero exposure time intensity (Figure 5E). For the erythrocyte sample, the ETDF is plotted from a single cell (circled in Figure A and B). The results show that ETDF enhancement of QDs conjugated with anti-band 3 antibodies in cells is greater than SWCNT-conjugated QDs, but it is still less than thin films of streptavidin QDs. The reduced ETDF enhancement is qualitatively consistent with previous results, suggesting that the band 3 proteins in nonparasitized erythrocytes exist both as oligomers and as monomers, as the QDs labeling monomeric band 3 proteins would not contribute to the ETDF enhancement. ${ }^{38}$ As oligomerization of band 3 proteins is known to be significantly increased in P. falciparuminfected and in sickle erythrocytes, a measurement of the monomer and oligomer population ratio will allow a quantitative measurement of the degree of band 3 clustering for quantitative diagnostics of malaria parasite infection or sickle cell disease in erythrocytes. To this end, we are working to establish measurement standards by investigating ETDF of QDs by systematically controlling the physical and chemical environments of clustered QDs, such as the length and charge of interparticle linker molecules and the number of QDs in a cluster.

\section{CONCLUSION}

We report the dynamic optical characteristics of single and clustered QDs, and QDs conjugated to a variety of materials. Under continuous exposure to an excitation light source, single QDs show quantized fluorescence emission fluctuation and quantized photo bleaching, but clustered QDs exhibit an increase in fluorescence emission at early exposure times. The rate and the total increase of emission from the clustered QDs depends upon the concentration of clustered QDs, the excitation power and exposure time, the presence of conjugated molecules on the QD surface, and QD conjugation to the substrate or other materials. Our results demonstrate that clustering of QDs coated with streptavidin proteins or antibodies enhances the fluorescence emission upon photo excitation, while the enhancement in QDs conjugated with metallic or low-band gap semiconductor materials, such as SWCNTs, is less significant. ETDF enhancement was also observed from QD-labeled band 3 proteins in human erythrocytes, which is indicative of the membrane protein clustering.

\section{ACKNOWLEDGEMENTS}

The authors give special thanks to the late Dr James A. Dvorak for his scientific dedication to malaria research at National Institute of Allergy and Infectious Diseases/National Institutes of Health (NIAID/NIH). The authors thank Dr Jeffrey Fagan for kindly providing DNA-wrapped SWCNTs and Dr Thomas E. Wellems at NIAID/NIH, Georgeta Crivat, Grace S. Chou, John Woodward, Garnett Bryant, and Paul DeRose at National Institute of Standards and Technology (NIST), and Dr Anil Patri at the Nanotechnology Characterization Laboratory (NCL) at SAIC-Frederick/NCI-Frederick for useful discussions. JH was supported by the NIST Advanced Technology Program. FT was supported by the Intramural Research Program of the NIAID/NIH. Official contribution of the NIST; not subject to copyright in the Unites States. Certain commercial equipment, instruments, or materials are identified in this article to foster understanding and does not imply recommendation or endorsement by NIST, it does not imply that the materials or equipment identified are necessarily the best available for the purpose. 


\section{REFERENCES}

1. Chan WCW, Nie SM. Quantum dot bioconjugates for ultrasensitive nonisotopic detection. Science 1998, 281:2016-2018.

2. Michalet X, Pinaud FF, Bentolila LA, Tsay JM, Doose $\mathrm{S}$, et al. Quantum dots for live cells, in vivo imaging, and diagnostics. Science 2005, 307:538-544.

3. Bruchez M, Moronne M, Gin P, Weiss S, Alivisatos AP. Semiconductor nanocrystals as fluorescent biological labels. Science 1998, 281:2013-2016.

4. Dubertret B, Skourides P, Norris DJ, Noireaux V, Brivanlou AH. et al. In vivo imaging of quantum dots encapsulated in phospholipid micelles. Science 2002, 298:1759-1762.

5. Edgar R, McKinstry M, Hwang J, Oppenheim AB, Fekete RA, et al. High-sensitivity bacterial detection using biotin-tagged phage and quantum-dot nanocomplexes. Proceedings of the National Academy of Sciences of the United States of America 2006, 103:4841-4845.

6. Krogmeier JR, Kang H, Clarke ML, Yim P, Hwang J. Probing the dynamic fluorescence properties of single water-soluble quantum dots. Optics Communications 2008, 281:1781-1788.

7. Anikeeva PO, Madigan CF, Coe-Sullivan SA, Steckel JS, Bawendi MG, et al. Photoluminescence of CdSe/ZnS core/shell quantum dots enhanced by energy transfer from a phosphorescent donor. Chemical Physics Letters 2006, 424:120-125.

8. Dwarakanath S, Bruno JG, Shastry A, Phillips T, John AA, et al. Quantum dot-antibody and aptamer conjugates shift fluorescence upon binding bacteria. Biochem Biophys Res Commun 2004, 325:739-743.

9. Medintz IL, Mattoussi H, Clapp AR, Goldman ER, Mauro JM. Prototype quantum dot FRET-based nanoscale biosensor. Abstracts of Papers of the American Chemical Society 2003, 226:U479-U480.

10. Clapp AR, Medintz IL, Uyeda HT, Fisher BR, Goldman ER, et al. Quantum dot-based multiplexed fluorescence resonance energy transfer. Journal of the American Chemical Society 2005, 127:18212-18221.

11. Zhang XQ, Guo Q, Cui DX. Recent advances in nanotechnology applied to biosensors. Sensors 2009, 9:1033-1053.

12. Chen Z, Li G, Zhang L, Jiang JF, Li Z, et al. A new method for the detection of ATP using a quantum-dottagged aptamer. Analytical and Bioanalytical Chemistry 2008, 392:1185-1188.

13. Snee PT, Somers RC, Nair G, Zimmer JP, Bawendi $\mathrm{MG}$, et al. A ratiometric $\mathrm{CdSe} / \mathrm{ZnS}$ nanocrystal $\mathrm{pH}$ sensor. Journal of the American Chemical Society 2006, 128:13320-13321.

14. Hohng S, Ha T. Near-complete suppression of quantum dot blinking in ambient conditions. Journal of the American Chemical Society 2004, 126:1324-1325.
15. Tsai CL, Tseng RJ, Yang Y, Ozkan CS. Quantum dot functionalized one dimensional virus templates for nanoelectronics. Journal of Nanoelectronics and Optoelectronics 2008, 3:133-136.

16. Biju V, Itoh T, Baba Y, Ishikawa M. Quenching of photoluminescence in conjugates of quantum dots and single-walled carbon nanotube. Journal of Physical Chemistry B 2006, 110:26068-26074.

17. Ishii S, Ueji R, Nakanishi S, Yoshida Y, Nagata H, et al. Fabrication of a quantum dot-polymer matrix by layer-by-layer conjugation. Journal of Photochemistry and Photobiology A: Chemistry 2006, 183:285-291.

18. Astefanoaei I, Dumitru I, Grimberg R, Stancu A. The effect of a metallic layer on energetic states of quantum dots. Sensor Letters 2007, 5:185-188.

19. Shavel A, Gaponik N, Eychmuller A. The assembling of semiconductor nanocrystals. European Journal of Inorganic Chemistry 2005, 19:3613-3623.

20. Ramachandran S, Merrill NE, Blick RH, van der Weide DW. Colloidal quantum dots initiating current bursts in lipid bilayers. Biosensors and Bioelectronics 2005, 20:2173-2176.

21. Xu CJ, Xing BG, Rao HH. A self-assembled quantum dot probe for detecting beta-lactamase activity. Biochemical and Biophysical Research Communications 2006, 344:931-935.

22. Sapsford KE, Pons T, Medintz IL, Mattoussi H. Biosensing with luminescent semiconductor quantum dots. Sensors 2006, 6:925-953.

23. Fagan JA, Landi BJ, Mandelbaum I, Simpson JR, Bajpai $\mathrm{V}$, et al. Comparative measures of single-wall carbon nanotube dispersion. Journal of Physical Chemistry B 2006, 110:23801-23805.

24. Fagan JA, Simpson JR, Landi BJ, Richter LJ, Mandelbaum I, et al. Dielectric response of aligned semiconducting single-wall nanotubes. Physical Review Letters 2007, 98:147402-147404.

25. Banerjee S, Wong SS. In situ quantum dot growth on multiwalled carbon nanotubes. Journal of the American Chemical Society 2003, 125:10342-10350.

26. Banerjee S, Wong SS. In situ growth of "fused", ozonized single-walled carbon nanotube-CdTe quantum dot junctions. Advanced Materials 2004, 16:34.

27. Zhou Z, Kang H, Clarke ML, Laceda SHDP, Zhao $\mathrm{M}$, et al. Water soluble DNA-wrapped single-wall carbon nanotube/quantum dot complexes. Small 2009, 5:2149-2155.

28. Tokumasu F, Dvorak J. Development and application of quantum dots for immunocytochemistry of human erythrocytes. Journal of Microscopy: Oxford 2003, 211:256-261.

29. Kuno M, Fromm DP, Hamann HF, Gallagher A, Nesbitt DJ. Nonexponential "blinking” kinetics of single 
CdSe quantum dots: A universal power law behavior. Journal of Chemical Physics 2000, 112:3117-3120.

30. Kuno M, Fromm DP, Gallagher A, Nesbitt DJ, Micic OI, et al. Fluorescence intermittency in single InP quantum dots. Nano Letters 2001, 1:557-564.

31. Kuno M, Fromm DP, Hamann HF, Gallagher A, Nesbitt DJ. "On"/“off" fluorescence intermittency of single semiconductor quantum dots. Journal of Chemical Physics 2001, 115:1028-1040.

32. Zhang Y, He J, Wang PN, Chen JY, Lu ZJ, et al. Time-dependent photoluminescence blue shift of the quantum dots in living cells: effect of oxidation by singlet oxygen. Journal of the American Chemical Society 2006, 128:13396-13401.

33. Jones M, Nedeljkovic J, Ellingson RJ, Nozik AJ, Rumbles G. Photoenhancement of luminescence in colloidal CdSe quantum dot solutions. Journal of Physical Chemistry B 2003, 107:11346-11352.
34. Chen HH, Leong KW. Quantum-dots-FRET nanosensors for detecting unamplified nucleic acids by single molecule detection. Nanomedicine 2006, 1:119-122.

35. Hohng S, Ha T. Single-molecule quantum-dot fluorescence resonance energy transfer. Chemphyschem 2005, 6:956-960.

36. Clapp AR, Medintz IL, Mattoussi H. Forster resonance energy transfer investigations using quantum-dot fluorophores. Chemphyschem 2006, 7:47-57.

37. Karachevtseva VA, Glamazda AY, DettlaffWeglikowska U, Kurnosov VS, Obraztsova ED, et al. Raman spectroscopy of HiPCO single-walled carbon nanotubes at 300 and 5 K. Carbon 2003, 41:1567-1574.

38. Tokumasu F, Fairhurst RM, Ostera GR, Brittain NJ, Hwang J, et al. Band 3 modifications in Plasmodium falciparum-infected AA and CC erythrocytes assayed by autocorrelation analysis using quantum dots. Journal of Cell Science 2005, 118:1091-1098.

\section{FURTHER READING}

Bryant G, Solomon G. Optics of Quantum Dots and Wires. Norwood: Artech House; 2005. 